\section{BMJ Open Respiratory Research}

\title{
Critical analysis of the utility of initial pleural aspiration in the diagnosis and management of suspected malignant pleural effusion
}

\author{
Rachel Mary Mercer (D , , ${ }^{1,2,3}$ Rebecca Varatharajah, ${ }^{1}$ Gillian Shepherd, ${ }^{1}$ Qiang Lu, ${ }^{1}$ \\ Olalla Castro-Añón, ${ }^{1,4}$ David J McCracken (D) , ${ }^{1}$ Alexandra Dudina, ${ }^{1}$ \\ Dinesh Addala, ${ }^{1}$ Stamatoula Tsikrika, ${ }^{1,5}$ Vineeth George (1) , ${ }^{1}$ Radhika Banka, ${ }^{1}$ \\ Rachelle Asciak, ${ }^{1}$ Maged Hassan (1) ${ }^{1,6}$ Robert Hallifax, ${ }^{1}$ Eihab O Bedawi, ${ }^{1}$ \\ Janis Kay Shute, ${ }^{2}$ Najib M Rahman ${ }^{1,3,7}$
}

To cite: Mercer RM, Varatharajah R, Shepherd G, et al. Critical analysis of the utility of initial pleural aspiration in the diagnosis and management of suspected malignant pleural effusion. BMJ Open Resp Res 2020;7:e000701. doi:10.1136/ bmjresp-2020-000701

- Additional material is published online only. To view, please visit the journal online (http://dx.doi.org/10. 1136/bmjresp-2020-000701).

Received 30 June 2020 Revised 4 August 2020 Accepted 10 August 2020
Check for updates

\section{(C) Author(s) (or their} employer(s)) 2020. Re-use permitted under CC BY-NC. No commercial re-use. See rights and permissions. Published by BMJ.

For numbered affiliations see end of article.

Correspondence to Dr Rachel Mary Mercer; rachel.mercer@nhs.net

\section{ABSTRACT}

Introduction Current guidelines recommend an initial pleural aspiration in the investigation and management of suspected malignant pleural effusions (MPEs) with the aim of establishing a diagnosis, identifying non-expansile lung (NEL) and, at times, providing a therapeutic procedure. A wealth of research has been published since the guidelines suggesting that results and outcomes from an aspiration may not always provide sufficient information to guide management. It is important to establish the validity of these findings in a 'real world' population.

Methods A retrospective analysis was conducted of all patients who underwent pleural fluid (PF) sampling, in a single centre, over 3 years to determine the utility of the initial aspiration.

Results A diagnosis of MPE was confirmed in 230/998 (23\%) cases, a further $95 / 998(9.5 \%)$ were presumed to represent MPE. Transudative biochemistry was found in 3\% of cases of confirmed MPE. Positive PF cytology was only sufficient to guide management in 45/140 (32\%) cases. Evidence of pleural thickening on CT was associated with both negative cytology $\left(\chi^{2} 1 \mathrm{df}=26.27, p<0.001\right)$ and insufficient samples $\left(\chi^{2} 1 \mathrm{df}=10.39, p=0.001\right)$. In NEL $44.4 \%$ of patients did not require further procedures after pleurodesis compared with $72.7 \%$ of those with expansile lung $\left(\chi^{2} 1 \mathrm{df}=5.49, p=0.019\right)$. In patients who required a combined diagnostic and therapeutic aspiration 106/113 (93.8\%) required further pleural procedures.

Conclusions An initial pleural aspiration does not achieve either definitive diagnosis or therapy in the majority of patients. A new pathway prioritising symptom management while reducing procedures should be considered.

\section{INTRODUCTION}

Pleural effusions are a common entity, and although around a third are due to malignancy, ${ }^{1}$ there is a wide differential diagnosis. The British Thoracic Society (BTS) Pleural Disease guidelines recommend initial pleural aspiration, ${ }^{2}$ often of large volume to

\section{Key messages}

In the modern era, the role of a pleural aspiration in the investigation and management of a suspected malignant pleural effusion should be re-evaluated.

It may be possible to triage some patients straight to a definitive procedure, rather than a pleural aspiration, but further assessment of the applicability and utility of this approach is needed before firm conclusions can be drawn.

- This study reviews the recently published trial evidence and tests their conclusions in a real-world population, and suggests that a review of the current pleural pathway should be undertaken.

relieve breathlessness, before proceeding to further investigations and potentially definitive treatments. Aspiration is recommended when a malignant pleural effusion (MPE) is suspected in order to obtain biochemical parameters (which may identify alternative diagnoses), provide cytology to confirm diagnosis and guide oncological treatment, and to identify both non-expansile lung (NEL) and those who experience symptomatic benefit, informing further pleural management choices.

Pleural fluid (PF) biochemistry allows the differentiation of transudate and exudate and identifies pleural inflammation. Although MPE is commonly considered to be exclusively exudative, case series have demonstrated transudates in $5 \%$ of cases. ${ }^{3}$ Biochemical parameters do not reliably differentiate between pleural infection and inflammatory MPE, with high lactate dehydrogenase (LDH), low glucose and low $\mathrm{pH}$ well recognised in either diagnosis. ${ }^{4}$ 
PF cytology has a diagnostic sensitivity of around $60 \%{ }^{2}$; however, with the expansion of personalised oncological treatments which require specific molecular testing, insufficient information may be derived from a single cytology sample. Tsim et a $\bar{l}$ demonstrated that testing was 'incomplete' (ie, insufficient material to permit molecular testing) in $47 \%$ of cytology positive effusions. In patients with a CT scan suggestive of malignancy, the OR for a malignant diagnosis with negative cytology was 2.25, but specific malignant radiological features were not assessed. ${ }^{5}$

Large volume aspiration may reveal the presence of NEL, where insertion of an indwelling pleural catheter (IPC) is preferable to attempting pleurodesis as definitive pleural management. ${ }^{6}$ Therapeutic aspiration confirms symptomatic benefit from the pleural procedure. Psallidas et $a l^{7}$ showed that $85.7 \%$ of patients experienced a clinically significant symptomatic benefit after fluid drainage but it was not possible to predict which patients would not experience symptomatic relief.

The current BTS pathway ${ }^{8}$ was proposed at a time where IPCs were considered second line treatment, and where oncological treatment options were not directed by molecular markers. As the focus of treatment has moved towards personalised treatment and patientrelated outcomes, and new data on molecular diagnostics and the value of biochemistry is available, the utility of initial pleural aspiration in the diagnostic pathway requires reassessment.

The aim of this study was to establish the utility of initial pleural aspiration in the diagnostic or management pathway of patients subsequently diagnosed with MPE. Specific areas addressed were: (1) if the presence of transudative biochemistry ruled out MPE; (2) the frequency with which malignant initial cytology materially altered management; (3) whether initial therapeutic aspiration reliably identifies NEL which influences management; (4) whether a single therapeutic aspiration prevented the need for further pleural procedures.

\section{METHODS}

Retrospective analysis was conducted of all inpatients and outpatients in our hospital where the first sample of PF obtained was between 1 January 2015 and 31 December 2017. If a previous sample was recorded greater than 6 months prior to the start of the study, the patient was still considered to represent a new case. Cases were identified through procedure records as, in our centre, all procedures (except in emergencies) are associated with an electronic report on either the radiology or respiratory documentation systems.

Data were collected from electronic patient records (EPR) by two separate physicians and any disagreements checked by a third physician. The EPR included laboratory results, procedure reports, need for further ipsilateral pleural procedure, radiology, clinic letters, multidisciplinary team outcomes and discharge reports, which were reviewed to determine treatment plans. Data were included until patient death or censorship (1 January 2019). Patients were excluded from specific analyses where information regarding requirement for further investigations, pleural procedures, treatment or date of death were unavailable. Drainage of over $200 \mathrm{~mL}$ fluid was considered to represent a therapeutic procedure.

CT reports, at the time of the aspiration, were reviewed to document radiological features of malignant pleural disease. ${ }^{9}$ It was assumed that radiological features were not present if no mention was made in the report. Reports were used, rather than independent reviews of each scan, with the aim of providing information relevant to a 'real-world' population.

The EPR was used to determine whether patients developed malignancy in the year after initial PF sampling was undertaken, to ensure cytology negative MPE were included. Follow-up of 12 months was considered sufficient to establish a malignant diagnosis, as per previous publications. $^{10}$

\section{Definitions}

Transudative effusions were identified using a cut-off $\mathrm{PF}$ protein $<30 \mathrm{~g} / \mathrm{L}$ and $\mathrm{LDH}<167 \mathrm{IU} / \mathrm{L}$ suitable to the normal ranges in our hospital. MPE was defined as either malignant cells in PF or malignant tissue in biopsy samples.

Patients were considered to have 'confirmed MPE' when there were malignant cells in either the PF or pleural biopsies. A broader definition of MPE (presumed MPE) was used for the NEL assessments due to the small number of patients with NEL, which required a larger sample size to draw meaningful conclusions. This included patients with pleural effusion in the context of histologically proven cancer elsewhere, excluding those with clear alterative causes,${ }^{11}$ or a radiologically confident diagnosis of MPE.

A PF sample was considered sufficient to guide management when it provided enough information to allow treatment decisions on systemic anticancer treatments to be made. Where no treatment was considered appropriate (eg, in the presence of poor performance status), positive cytology leading to the decision not to treat was considered sufficient. Cases where further biopsies beyond initial pleural aspiration were needed to determine precise anticancer therapy were deemed to be insufficient. Cases in which a concurrent biopsy was taken at the time of the first PF aspiration were excluded from the diagnostic analysis.

The presence of NEL was defined pre-hoc and scored using the following criteria by two respiratory physicians independently. 'Probable' and 'proven' non-expansile definitions as below were considered as NEL:

- Proven expansile: fully expanded lung, clear visualisation of the costophrenic angle and no evidence of fluid or air within the pleural cavity. 
Table 1 Type of cancer causing MPE and method of diagnosis

\begin{tabular}{|c|c|c|c|c|c|c|}
\hline \multirow[b]{2}{*}{ Type of cancer } & \multicolumn{2}{|c|}{ Confirmed MPE } & \multicolumn{2}{|c|}{ Presumed MPE } & \multirow[b]{2}{*}{ Total } & \multirow[b]{2}{*}{$\begin{array}{l}\text { Confirmed versus } \\
\text { presumed significance }\end{array}$} \\
\hline & $\begin{array}{l}\text { Malignant } \\
\text { cytology }\end{array}$ & $\begin{array}{l}\text { Malignant } \\
\text { pleural } \\
\text { biopsy }\end{array}$ & $\begin{array}{l}\text { Histological } \\
\text { malignancy } \\
\text { elsewhere }\end{array}$ & $\begin{array}{l}\text { Clinical } \\
\text { diagnosis }\end{array}$ & & \\
\hline Breast & 49 (84.5\%) & $5(8.6 \%)$ & $2(3.4 \%)$ & $2(3.4 \%)$ & 58 & $p<0.001$ \\
\hline NSCLC & 44 (55.7\%) & $11(13.9 \%)$ & $18(22.8 \%)$ & $6(7.6 \%)$ & 79 & $p=0.78$ \\
\hline SCLC & $4(80 \%)$ & $0(0 \%)$ & $1(20 \%)$ & $0(0 \%)$ & 5 & $p=0.65$ \\
\hline Mesothelioma & $3(6.3 \%)$ & 42 (87.5\%) & $1(2.1 \%)$ & $2(4.2 \%)$ & 48 & $\mathrm{p}<0.001$ \\
\hline Gynaecological & $21(75.0 \%)$ & $0(0 \%)$ & 7 (25.0\%) & $0(0 \%)$ & 28 & $p=0.67$ \\
\hline CUP & $7(41.2 \%)$ & $2(11.8 \%)$ & $6(35.3 \%)$ & $2(11.8 \%)$ & 17 & $p=0.11$ \\
\hline Lower Gl & $6(37.5 \%)$ & $0(0 \%)$ & 9 (56.3\%) & $1(6.3)$ & 16 & $p=0.008$ \\
\hline Upper Gl & 5 (50.0\%) & 2 (20.0\%) & 3 (30.0\%) & $0(0 \%)$ & 10 & $p=1.00$ \\
\hline Lymphoma & $6(37.5 \%)$ & $3(18.8 \%)$ & 7 (43.8\%) & $0(0 \%)$ & 16 & $p=0.26$ \\
\hline Melanoma & 5 (83.3\%) & $0(0 \%)$ & $1(16.7 \%)$ & $0(0 \%)$ & 6 & $p=0.68$ \\
\hline Pancreatic & $2(40.0 \%)$ & $0(0 \%)$ & $0(0 \%)$ & $3(60.0 \%)$ & 5 & $p=0.15$ \\
\hline Urological & $4(40.0 \%)$ & $1(10.0 \%)$ & 5 (50.0\%) & $0(0 \%)$ & 10 & $p=0.16$ \\
\hline Head and neck & $0(0 \%)$ & $1(20.0 \%)$ & 4 (80.0\%) & $0(0 \%)$ & 5 & $p=0.03$ \\
\hline Sarcoma & $0(0 \%)$ & $0(0 \%)$ & $6(100 \%)$ & $0(0 \%)$ & 6 & $p<0.001$ \\
\hline Other & $6(54.5 \%)$ & $1(9.1 \%)$ & $4(36.4 \%)$ & $0(0 \%)$ & 11 & $p=0.74$ \\
\hline Unclear & $0(0 \%)$ & $0(0 \%)$ & 2 (40.0\%) & $3(60.0 \%)$ & 5 & $p=0.002$ \\
\hline Total & $162(49.8 \%)$ & 68 (20.9\%) & 76 (23.4\%) & 19 (5.8\%) & 325 & \\
\hline
\end{tabular}

Fishers exact test used for all calculations.

CUP, cancer of unknown primary; GI, gastrointestinal; MPE, malignant pleural effusion; NSCLC, non-small cell lung cancer; SCLC, small cell lung cancer.

- No evidence of non-expansile: no evidence of trapping (or air) in the pleural cavity but residual fluid.

- Probable non-expansile: small volume of air in the pleural cavity and/or evidence of visceral pleural thickening and pattern of fluid which would not be consistent with normal gravitational distribution.

- Proven non-expansile: clear evidence of air in the pleural cavity preventing, either partial of complete, apposition of the visceral pleural to the haemithorax.

\section{Patient and public involvement statement}

Patients and the public were not involved in the research.

\section{RESULTS}

Over the study period, 998 patients underwent $\mathrm{PF}$ aspiration with multiple diagnoses (see appendix 1). In total, 325/998 patients (32.6\%) were diagnosed with MPE by any definition, and 230/325 (70.1\%) of these had malignant cells in PF or tissue samples. Table 1 provides details on the underlying malignancy.

\section{Transudative effusions}

In total, 223 patients with confirmed MPE had samples send for biochemical analysis of which, 7/223 $(3.14 \%$ $95 \%$ CI $0.9 \%$ to $5.4 \%$ ) had biochemistry consistent with transudate. Of these, the median $\mathrm{LDH}$ was $117 \mathrm{IU} / \mathrm{L}$
(IQR 80-136) and protein was 25 g/L (IQR 20-28), two were systemically unwell, two had liver disease, one had congestive cardiac failure and two patients did not have any underlying diseases to suggest a transudative pathology.

\section{Utility of the initial aspiration sample or radiology to guide management}

In 152 patients $(46.7 \%)$, cytological examination was requested to provide diagnostic information to guide management. Twelve underwent concurrent pleural biopsies and were excluded, leaving 140 patients. Of these 140 patients, 78 (55.7\%) had positive cytology (71 on first aspiration) and this information was sufficient to guide management in $45 / 71(63.4 \%)$ of cytology positive cases. The initial aspiration was sufficient to guide management in $45 / 140(32.1 \%)$ of the total number of MPE cases (see table 2 for details).

Baseline clinical (demographic) and radiological features were assessed to determine predictors of positive cytology, and sufficiency to guide management (tables 3 and 4). Contemporaneous CT scans were available in 135 cases of confirmed MPE. Pleural thickening and nodularity were negatively associated with positive PF cytology on initial sampling whereas the presence of lymphangitis was associated with positive PF cytology. 


\begin{tabular}{lr}
\hline Table 2 & Details of treatment decisions \\
\hline Treatment decision & Patients \\
\hline Diagnostic only - not fit for SACT & $7(15.6 \%)$ \\
\hline SACT options not suitable for patient & $12(26.7 \%)$ \\
\hline Immunotherapy or hormonal therapy initiated & $9(20.0 \%)$ \\
Chemotherapy initiated & $13(28.9 \%)$ \\
SACT altered in response to results & $1(2.2 \%)$ \\
Patient refused treatment & $3(6.6 \%)$ \\
\hline
\end{tabular}

SACT, systemic anti cancer treatment.

Due to the known low sensitivity of fluid cytology in the diagnosis of malignant mesothelioma, ${ }^{12}$ the analysis was repeated excluding mesothelioma cases $(n=43)$. The negative predictive value of pleural thickening for cytology positivity remained $\left(\chi^{2} 1 \mathrm{df}=11.66, \mathrm{p}=0.001\right)$.

\section{Non-expansile lung}

Radiology post initial aspiration of sufficient quality to identify NEL was available in 318/325 patients $(97.8 \%)$. Probable or proven NEL was found in 64/318 (20.1\%) patients; in 38/318 (11.9\%), at least one reviewer reported proven NEL but both initial reviewers only agreed there was proven NEL in 19/318 (6\%) of cases.

Therapeutic aspiration with a chest radiograph within the next 24 hours was conducted in 153/325 (47.1\%, mean drainage $1114 \mathrm{~mL}$ SD 458). After initial aspiration radiograph results, pleurodesis was not attempted due to evidence of NEL in 7/153 (4.6\%) cases. In one case, the lung was later shown to be expansile but was nonexpansile in the remaining 6 . The sensitivity and specificity of the initial post aspiration chest radiograph for the diagnosis of NEL was $24.0 \%$ (95\% CI $9.4 \%$ to $45.1 \%$ ) and $99.2 \%$ (95\% CI $95.7 \%$ to $100.0 \%$ ).

Of 106 patients who underwent pleurodesis, 18 radiographs were classified as NEL, 1 of whom had evidence of NEL prior to a diagnostic thoracoscopy, but an attempt at pleurodesis was considered appropriate. 8/18 (44.4\%) patients with NEL did not require any further pleural procedures at 3 months (or until death) compared with $64 / 88(72.7 \%)$ in the patients with no evidence of NEL $\left(\chi^{2} 1 \mathrm{df}=5.49, \mathrm{p}=0.019\right)$. When 10 patients in the probable NEL group were compared with those without evidence of NEL, there was still a significantly higher requirement for subsequent procedures $\left(\chi^{2} 1 \mathrm{df}=4.53, \mathrm{p}=0.033\right)$.

\section{Single therapeutic aspiration only}

In total, $7 / 113(6.2 \%)$ patients who underwent a combined diagnostic and therapeutic procedure did not require any further pleural interventions for fluid control. Of patients who only required one aspiration, $4 / 7(57.1 \%)$ died within 30 days compared with 5/106 $(4.7 \%)$ of those who required more than one procedure $\left(\chi^{2} 1 \mathrm{df}=24.62, \mathrm{p}<0.0001\right)$.

\section{DISCUSSION}

The currently used pathway for investigation of potential MPE has been unchanged for over 15 years, despite new evidence on cytological accuracy, requirement for molecular markers and no modern re-assessment of the utility of biochemical results. Pleural aspiration as an initial diagnostic step is promoted based on being less invasive than pleural biopsy techniques, allowing diagnosis of NEL and providing important information including 'diagnostic' cytology and the presence of transudate. In this study, we adopted a pragmatic approach to assess if the current BTS pathway remains relevant to modern practice and to ensure that recently published study results are applicable to real-world practice.

In the present study, $3 \%$ of those with confirmed MPE had transudative biochemistry, which is consistent with previously published data. ${ }^{3}$ It is widely recognised that $\mathrm{pH}$ and glucose may be low in both MPE and many other pathologies. ${ }^{4}$ These data suggest that biochemical results alone are insufficient to completely exclude MPE as a cause of an undiagnosed effusion.

Sensitivity of $\mathrm{PF}$ for cytological diagnosis is between $46 \%$ and $67 \%$ for patients with MPE, ${ }^{13} 14$ although a recent study showed that it was not possible to identify all the 'necessary predictive markers' in $47 \%$ of cases. ${ }^{5}$ The aim of the present study was to use a pragmatic approach to determine the utility of the information gleaned from PF cytology, that is, whether the information was sufficient to guide ongoing management. A positive management decision covered a spectrum of outcomes from palliation at one end to initiation of mutation driven oncological therapies at the other. Despite this broad

Table 3 Positive cytology related to gender and CT scan features

\begin{tabular}{llcl}
\hline & \multicolumn{2}{l}{ Cytology positive in initial pleural fluid sample } & \multicolumn{1}{l}{ Significance } \\
\cline { 2 - 3 } & Yes & No & $\chi^{2} 1 \mathrm{df}=15.34, p<0.001$ \\
\hline Male gender & $27 / 71(38 \%)$ & $49 / 69(71 \%)$ & $\chi^{2} 1 \mathrm{df}=26.27, p<0.001$ \\
Pleural thickening & $29 / 68(43 \%)$ & $57 / 67(85 \%)$ & $\chi^{2} 1 \mathrm{df}=9.11, p=0.003$ \\
\hline Pleural nodularity & $22 / 68(32 \%)$ & $39 / 67(58 \%)$ & $\chi^{2} 1 \mathrm{df}=7.81, p=0.005$ \\
\hline Lymphangitis & $12 / 68(18 \%)$ & $2 / 67(3 \%)$ & $\chi^{2} 1 \mathrm{df}=1.12, p=0.290$ \\
\hline Pericardial effusion & $3 / 68(4 \%)$ & $6 / 67(9 \%)$ & $\chi^{2} 1 \mathrm{df}=1.25, p=0.263$ \\
\hline Thoracic lymphadenopathy & $37 / 68(54 \%)$ & $30 / 67(45 \%)$ & \\
\hline
\end{tabular}


Table 4 Actionable cytology related to gender and CT scan features

\begin{tabular}{|c|c|c|c|}
\hline & Sample suff & nanagement & \\
\hline & Yes & No & Significance \\
\hline Male gender & $20 / 45(44 \%)$ & $56 / 95(59 \%)$ & $\chi^{2} 1 \mathrm{df}=2.58, p=0.108$ \\
\hline Pleural thickening & $19 / 43(44 \%)$ & $68 / 92(74 \%)$ & $\chi^{2} 1 \mathrm{df}=10.39, p=0.001$ \\
\hline Pleural nodularity & $13 / 43(30 \%)$ & 49/92 (53\%) & $\chi^{2} 1 \mathrm{df}=5.70, p=0.017$ \\
\hline Lymphangitis & 9/43 (21\%) & $5 / 92(5 \%)$ & $\chi^{2} 1 d f=7.57, p=0.006$ \\
\hline Pericardial effusion & $4 / 43(9 \%)$ & $5 / 92(5 \%)$ & $\chi^{2} 1 d f=0.74, p=0.401$ \\
\hline Thoracic lymphadenopathy & $27 / 43(63 \%)$ & 40/92 (43\%) & $\chi^{2} 1 d f=4.37, p=0.037$ \\
\hline
\end{tabular}

range of acceptable outcomes, an initial pleural aspiration provided sufficient information to guide management in only $63 \%$ of patients who had initially positive PF cytology, and in $32 \%$ of cases of MPE as a whole. If the analysis also included patients with 'presumed' MPE, the success rate is likely to be even lower.

The majority of patients with MPE experience fluid recurrence and require definitive management of the pleural space. A suggested role of a large volume aspiration is to diagnose NEL, and thus to prevent patients undergoing pleurodesis which is likely to be unsuccessful. A recently published study showed that NEL is present in between $17 \%$ and $34 \%$ of MPE, but is difficult to diagnose on chest radiograph. ${ }^{15}$ In view of this finding, patients were categorised into 'proven' and 'probable' NEL to establish the incidence of both types of NEL, the ability to detect NEL after one aspiration and whether NEL affects pleurodesis success. The prevalence ranged from $6 \%$ to $20 \%$, but the sensitivity of the post procedure chest radiograph for detecting NEL was only $24 \%$. In patients who underwent pleurodesis there was a significantly higher requirement for further procedures, indicating treatment failure, in those diagnosed with NEL. This finding makes sense, as there is a lack of pleural apposition in NEL, but importantly, this difference was maintained even when those with probable NEL alone were analysed. This suggests that any suspicion of NEL on chest radiograph is likely to be associated with pleurodesis failure, but this is often not evident until after the first post procedure radiograph.

Out of a cohort of 113 patients who required both an initial diagnostic and therapeutic intervention only 7 (6\%) patients required a single aspiration, and only 3 of these patients survived over 30 days after the procedure.

There is a wealth of information which may now help to inform a 'new pleural pathway'. Our study confirms the findings from Tsim et al that CT evidence of malignancy was associated with negative cytology ${ }^{5}$; specifically the present study demonstrated that pleural thickening and nodularity are highly associated with negative PF cytology. Importantly, the significance of pleural thickening was maintained even when patients with mesothelioma (which could be a confounder considering the high rates of thickening and low cytology positivity) were excluded.
The CT finding of lymphangitis was associated with positive PF cytology-this could represent more advanced malignancy or relate to the manner in which tumours metastasise. Further studies with robust criteria to ensure that radiology reports are standardised are needed to validate these findings. If such features are consistently predictive, it may be possible to stratify patients to different initial diagnostic techniques according to the likelihood of obtaining sufficient cytology on the basis of the initial CT findings.

Another compelling argument for changing the current pathway is the burden of symptoms for the patients. Studies have shown that symptomatic benefits after a therapeutic aspiration are transient, and sometimes only last a matter of days. ${ }^{16}$ There is evidence to show that diagnostic uncertainty is detrimental to the patient's wellbeing ${ }^{17}$ and this, in combination with distressing symptoms, may be a consequence of the current prolonged pathway. Tsim et alshowed that the median time from first outpatient appointment (or emergency admission) to diagnosis was 26 days, which may not necessarily include the time to definitive management of the pleural space and therefore relief of symptoms. ${ }^{5}$ Further qualitative work is needed to map the patient experience of MPE to ensure that any revised pathway takes this into account.

A number of alternative diagnostic pathways have been suggested such as a 'straight to local anaesthetic thoracoscopy' approach for patients with a higher risk of mesothelioma (asbestos exposure and a suggestive CT scan). ${ }^{5}$ Non-invasive ultrasound methods such as speckle tracking and $\mathrm{M}$ mode ultrasound have a reported sensitivity of $71 \%$ and $50 \%$, respectively, with a specificity of $85 \%^{18}$ in identifying NEL and could be used to triage patients to IPC or pleurodesis. The PRE-EDIT (Elastase-Directed Intrapleural Catheter or Talc Pleurodesis) study trialled use of manometry to detect NEL and triage to pleurodesis or IPC, depending on pleural pressures. ${ }^{19}$ Bhatnagar et $a l^{20}$ proved that instilling talc via an IPC increases the spontaneous pleurodesis rate in MPE treated with IPC (to around 50\%). Despite all the above advances, a rational pathway combining all relevant information has yet to be produced.

On this basis of our data, it may be appropriate to adopt an approach which moves straight to a definitive procedure in a subset of cases. In a diagnostic setting, 
this could be straight to local anaesthetic thoracoscopy or image guided biopsy, especially in cases where it is well recognised that the cytological yield is low, such as in suspected mesothelioma. Likewise, in cases where there is a high suspicion of MPE and patient preference is for an IPC, it may be appropriate to perform IPC insertion as a first line management option. Removal of IPCs shortly after insertion is relatively uncomplicated due to the lack of adhesion formation and is therefore easily reversible if a non-MPE diagnosis is achieved, or if IPC treatment was not tolerated and outpatient pleurodesis can be undertaken through the IPC.

Further work is needed before these strategies are adopted into clinical practice. Risk stratification would need to be validated to establish which patients would benefit from an initial pleural biopsy, the relative complications of an IPC versus an aspiration would need to be established and importantly qualitative work would need to be undertaken to ensure acceptability to patients. Even if an alternative pathway was found to be beneficial, it would not necessarily be feasible to introduce on a national scale, due to differences in availability of procedures between hospitals. For centres with access to some, or all, of these procedures, clear evidence on the risks and benefits of a more tailored approach is critical to shared decision making with patients.

This study has significant limitations, mainly the retrospective nature which means that relevant, available data could have been missed. The diagnosis of a transudative effusion was made solely on the PF biochemistry, which is less reliable than the use of Light's criteria, but the findings from this cohort are consistent with other published literature. The decision regarding whether the PF cytology was sufficient to guide treatment was made on retrospective data, and there could have been variations in practice between clinicians. The radiology was not performed at the time of the aspiration so there may have been changes to the radiological features described when the aspiration was undertaken. Despite this, the real-world cohort is concordant with published literature within trial populations, which provides credibility to previous findings.

Further important work includes review of radiology for all patients who underwent aspiration to assess what proportion of CT scans reported a suspicion of malignancy which was later disproved. In a revised pathway with a 'straight to IPC' option, this would help to identify the number of patients who may have unnecessary IPC insertion, although many of these patients would still have pleural biopsies undertaken later on as part of the current pathway if negative PF cytology was obtained.

In conclusion, the current BTS pathway which suggests initial pleural aspiration for diagnostic and therapeutic purposes does not often achieve either definitive diagnosis or therapy and our study suggests a review of the current approach would be beneficial. Further research is required with a focus on patient reported outcomes, to provide a novel pathway which prioritises symptoms and minimises procedures in this patient population where the focus is on improving, rather than sustaining, life, but this must always take into account the ability of the pathway to be performed in a range of centres. Recent advances in cancer care and information on optimal pleural disease management may provide the opportunity to make a significant difference to the lives of patients with MPE.

Author affiliations

${ }^{1}$ Oxford Centre for Respiratory Medicine, Churchill Hospital, Oxford, United Kingdom

${ }^{2}$ School of Pharmacy and Biomedical Sciences, University of Portsmouth, Portsmouth, UK

${ }^{3}$ Oxford Respiratory Trials Unit, Churchill Hospital, Oxford, United Kingdom ${ }^{4}$ Respiratory Division and Sleep Disorders Unit, Lucus Augusti University Hospital, Lugo, Spain

${ }^{5}$ Pulmonary Division, Department of Critical Care, Medical School, National and Kapodistrian, University of Athens, Evangelismos General Hospital,

Athens, Greece

${ }^{6}$ Chest Diseases Department, Faculty of Medicine, Alexandria University, Alexandria, Egypt

${ }^{7}$ Oxford NIHR Biomedical Research Centre, University of Oxford and Oxford University Hospitals, Oxford, UK

Contributors RMM was responsible for initial draft preparation and revision. All authors were responsible for data collection, reviewing and approving the final manuscript. RMM and NMR are responsible for the overall content as guarantors.

Funding RH is funded by an NIHR Clinical Lectureship. NMR, MH and OC-A acknowledge the support of the European Respiratory Society Fellowships. NMR is supported by the NIHR Oxford Biomedical Research Centre.

Competing interests NMR reports grants and personal fees from Rocket Medical UK, grants from BD USA, personal fees from Lung therapeutics USA, outside the submitted work. VG reports personal fees from Teva UK Ltd/Haymarket Media Group, outside the submitted work.

Patient and public involvement Patients and/or the public were not involved in the design, or conduct, or reporting, or dissemination plans of this research.

Patient consent for publication Not required.

Ethics approval This research was approved by the Health Research Authority (IRAS project ID 275779); the need for informed consent was waived.

Provenance and peer review Not commissioned; externally peer reviewed.

Data availability statement No data are available.

Open access This is an open access article distributed in accordance with the Creative Commons Attribution Non Commercial (CC BY-NC 4.0) license, which permits others to distribute, remix, adapt, build upon this work non-commercially, and license their derivative works on different terms, provided the original work is properly cited, appropriate credit is given, any changes made indicated, and the use is non-commercial. See: http://creativecommons.org/licenses/by-nc/4.0/.

ORCID iDs

Rachel Mary Mercer http://orcid.org/0000-0003-3645-3151

David J McCracken http://orcid.org/0000-0002-8278-478X

Vineeth George http://orcid.org/0000-0001-6292-5875

Maged Hassan http://orcid.org/0000-0002-8768-6548

\section{REFERENCES}

1 Loveland P, Christie M, Hammerschlag G, et al. Diagnostic yield of pleural fluid cytology in malignant effusions: an Australian tertiary centre experience. Intern Med J 2018;48:1318-24.

2 Hooper C, Lee YCG, Maskell N, et al. Investigation of a unilateral pleural effusion in adults: British thoracic Society pleural disease guideline 2010. Thorax 2010;65(Suppl 2):ii4-17.

3 Ashchi M, Golish J, Eng P, et al. Transudative malignant pleural effusions: prevalence and mechanisms. South Med J 1998;91:23-6.

4 Porcel-Pérez JM, Vives Soto M, Esquerda Serrano A, et al. [Cuttoff values of biochemical tests on pleural fluid: their usefulness in 
differential diagnosis of 1,040 patients with pleural effusion]. An Med Interna 2004;21:113-7.

5 Tsim S, Paterson S, Cartwright D, et al. Baseline predictors of negative and incomplete pleural cytology in patients with suspected pleural malignancy - Data supporting 'Direct to LAT' in selected groups. Lung Cancer 2019;133:123-9.

6 Feller-Kopman DJ, Reddy CB, DeCamp MM, et al. Management of malignant pleural effusions. An official ATS/STS/STR clinical practice guideline. Am J Respir Crit Care Med 2018;198:839-49.

7 Psallidas I, Yousuf A, Talwar A, et al. Assessment of patient-reported outcome measures in pleural interventions. BMJ Open Respir Res 2017;4:e000171.

8 Roberts ME, Neville E, Berrisford RG, et al. Management of a malignant pleural effusion: British thoracic Society pleural disease guideline 2010. Thorax 2010;65(Suppl 2):ii32-40.

9 Leung AN, Müller NL, Miller RR. CT in differential diagnosis of diffuse pleural disease. AJR Am J Roentgenol 1990;154:487-92.

10 Janssen J, Maldonado F, Metintas M. What is the significance of non-specific pleuritis? A trick question. Clin Respir J 2018;12:2407-10.

11 Rahman NM, Pepperell J, Rehal S, et al. Effect of opioids vs NSAIDs and larger vs smaller chest tube size on pain control and pleurodesis efficacy among patients with malignant pleural effusion: the time1 randomized clinical trial. JAMA 2015;314:2641-53.

12 Woolhouse I, Bishop L, Darlison L, et al. BTS guideline for the investigation and management of malignant pleural mesothelioma. BMJ Open Respir Res 2018;5:e000266.
13 Lim MH, Garrettc J, Mowlem L, et al. Diagnosing malignant pleural effusions: how do we compare? N Z Med J 2013;126:42-8.

14 Arnold DT, De Fonseka D, Perry S, et al. Investigating unilateral pleural effusions: the role of cytology. Eur Respir J 2018;52. doi:10.1183/13993003.01254-2018. [Epub ahead of print: 08 Nov 2018].

15 Martin GA, Kidd AC, Tsim S, et al. Inter-Observer variation in image interpretation and the prognostic importance of non-expansile lung in malignant pleural effusion. Respirology 2020;25:298-304.

16 Boshuizen RC, Vincent AD, van den Heuvel MM. Comparison of modified Borg scale and visual analog scale dyspnea scores in predicting re-intervention after drainage of malignant pleural effusion. Support Care Cancer 2013;21:3109-16.

17 Risberg T, Sørbye SW, Norum J, et al. Diagnostic delay causes more psychological distress in female than in male cancer patients. Anticancer Res 1996;16:995-9.

18 Salamonsen MR, Lo AKC, Ng ACT, et al. Novel use of pleural ultrasound can identify malignant entrapped lung prior to effusion drainage. Chest 2014;146:1286-93.

19 Martin GA, Tsim S, Kidd AC, et al. Pre-EDIT: a randomized feasibility trial of Elastance-Directed intrapleural catheter or talc pleurodesis in malignant pleural effusion. Chest 2019;156:1204-13.

20 Bhatnagar R, Keenan EK, Morley AJ, et al. Outpatient talc administration by indwelling pleural catheter for malignant effusion. N Engl J Med 2018;378:1313-22. 